\title{
YPF-Tecnología (Y-TEC) y su rol en la política científico- tecnológica nacional
}

\section{Julián Bilmes}

Lic. en Sociología. Becario doctoral CONICET Instituto de Investigaciones en Humanidades y Ciencias Sociales -IdIHCS-( UNLP, CONICET). Centro de Investigaciones en Política y Economía

Resumen: Se analiza la política científicotecnológicade laEmpresa Nacional Yacimientos Petrolíferos Fiscales (YPF) a partir de su renacionalización parcial en 2012, tomando el caso de su brazo tecnológico, YPF-Tecnología (Y-TEC). Se indaga en su formato, concepción y actividades durante el último gobierno de Cristina Fernández de Kirchner (CFK) (20132015), bajo un paradigma mixto de gestión público-privada y profesionalizada. Atendiendo a las limitaciones de ese diseño, consistente con la estrategia de desarrollo de ese gobierno, se analiza luego el nuevo rol a cumplir por Y-TEC bajo el gobierno de Mauricio Macri (2016-2017). El paradigma de Innovación Abierta de Y-TEC en este último período, que se corresponde con el capitalismo contemporáneo, globalizado y "flexible", da cuenta, finalmente, de cómo este nuevo gobierno nacional se aprovechó del formato de Y-TEC, manteniendo la gestión, pero redefiniendo los proyectos originales, para capitalizar esa estructura bajo otros objetivos y un nuevo modelo, afín al gran capital.

Palabras clave: YPF-Tecnología (Y-TEC), innovación abierta, desarrollo tecnológico, capitalismo
YPF- Tecnología (Y-TEC) and its role in the national scientific-technological policy

Abstract: The scientific-technological policy of the National Company Yacimientos Petroliferos Fiscales (YPF) is analyzed from its partial renationalization in 2012, taking the case of its technological arm, YPF-Tecnología (Y-TEC). Its format, conception and activities during the last government of Cristina Fernández de Kirchner (CFK) (2013-2015) is discussed, under a mixed paradigm of public-private and professionalized management. Considering the limitations of this design, consistent with the development strategy of that government, the new role to be fulfilled by $\mathrm{Y}$-TEC under the government of Mauricio Macri (2016-2017) is then analyzed. The Y-TEC Open Innovation paradigm proposed by the new administration is consistent with the contemporary, globalized and "flexible" capitalism and reveals how this new national government took advantage of the original Y-TEC format. The new policy maintains the management, but redefines the original projects, to capitalize the structure under other objectives and a new model.

Key words: YPF-Technology (Y-TEC), open innovation, technological development, capitalism 


\section{Introducción}

En abril de 2012 la presidenta CFK anunciaba la intervención estatal en la histórica empresa petrolera nacional, YPF, junto con el envío a la Legislatura del proyecto de ley de "Soberanía Hidrocarburífera" (ley 26.741), que sería aprobada por amplia mayoría. Los principios de la ley daban cuenta de la necesidad de desarrollar una política científico-tecnológica (C-T) por parte del Estado nacional en pos de lograr el deseado autoabastecimiento energético.

En diciembre de 2012 se creó YPF-Tecnología (Y-TEC), en base a una asociación estratégica entre YPF y el Consejo Nacional de Investigaciones Científicas y Técnicas (CONICET), con la misión de brindar soluciones tecnológicas al sector energético y formar especialistas para el desarrollo de la industria de la región. YPF pasó a encargarse del gerenciamiento general, con el 51\% del paquete accionario, y el CONICET, con el restante 49\%, aportaría un plantel de investigadores, técnicos y becarios, junto con su red de institutos en todo el país. YPF aportó también su personal técnico, infraestructura y equipamiento, y transfirió su Dirección de Tecnología. El discurso oficial de Y-TEC caracterizaba como "un hito la revalorización del papel de la ciencia y la tecnología al servicio del desarrollo", y la vinculación entre YPF y CONICET era anunciada como "un hecho inédito y de alto impacto" para el país.

Este trabajo analiza esta experiencia, en el marco del objetivo de impulsar un modelo de l+D+i (Investigación, Desarrollo e Innovación) orientado a promover el desarrollo económico social del país, así como un intento de vinculación entre el Estado, el complejo C-T nacional y el sistema productivo. Se indaga entonces en su concepción, diseño y características, y en sus implicancias económico-sociales'.

\section{Rol, concepción y formato de Y-TEC}

El formato definido para Y-TEC implicaba un perfil novedoso, en sintonía con el nuevo paradigma mixto de gestión público-privada de YPF, el cual recuperaba elementos de los modelos previos (estatal y privado). Así, la Ley 26.741 contemplaba la expropiación del 51\% de las acciones de YPF, pertenecientes a Repsol, recuperando el Estado la dirección estratégica de la empresa, pero sosteniendo su condición de sociedad anónima, y definiéndose un modelo de gerenciamiento bajo "gestión profesionalizada". Fue así que se designó al frente de YPF a Miguel Galuccio, ex alto ejecutivo de Schlumberger, principal compañía de servicios hidrocarburíferos, y éste designó luego a otro ex Schlumberger, Bernard Gremillet, como Chief Technology Officer (CTO) de la empresa y a Gustavo Bianchi como Director de Tecnología (quien sería luego primer Director de Y-TEC). En este marco la principal función de Y-TEC consistía en desarrollar el know-how necesario para explotar los abundantes hidrocarburos "no convencionales" de Vaca Muerta², un papel estratégico para el proyecto político-económico del último kirchnerismo en pos de superar el déficit energético que jaqueaba

\footnotetext{
1 Se han recopilado datos de la página web de Y-TEC y su revista Desafíos, así como de diversos diarios, portales y revistas especializadas. Se agradece la orientación brindada por Alberto Keitelman, trabajador de Y-TEC desde 2013 hasta 2015, quien gentilmente accedió a ser entrevistado en profundidad para este trabajo.

${ }^{2}$ Formación considerada como el segundo mayor reservorio mundial de gas no convencional y cuarto en petróleo no convencional, lo cual ubica a nuestro país en el tercer lugar mundial en estos recursos.
} 
el modelo de acumulación neodesarrollista en curso (Bilmes, 2017). Se apuntaba incluso a ser líderes mundiales en I+D en ese tipo de recursos.

La original articulación YPF-CONICET buscaba generar una sinergia de conocimientos, experiencias y capacidades de ambas partes, así como potenciar y optimizar el rendimiento del personal y los recursos a disposición. Bajo una concepción heredera de las ideas de Jorge Sábato y su famoso "triángulo de relaciones científico-tecnológicas", se buscaba, desde el gobierno, acoplar la infraestructura C-T (que el proyecto kirchnerista había vigorizado con gran cantidad de recursos) a las demandas de la estructura productiva, con el objetivo de potenciar el desarrollo mediante la innovación.

Así, desde un primer momento se buscó articular con instituciones del complejo C-T de nuestro país, así como también con las universidades que realizaran I+D y estuvieran en condiciones de aportar valor agregado. La cantidad de tecnólogos trabajando en Y-TEC, por otro lado, no detuvo su crecimiento: desde 80 en sus inicios a más de 300 en la actualidad, entre investigadores, técnicos, becarios y consultores. Se desarrolló toda una serie de políticas tendientes a convocar investigadores, mediante financiamiento de proyectos de investigación orientados y diversas becas otorgadas por la Fundación YPF en conjunto con programas estatales, junto con las convocatorias a investigadores del CONICET para integrarse en proyectos de I+D de Y-TEC, la cual pasaría a funcionar como una unidad de vinculación del organismo estatal.

La gran apuesta que comportaba este emprendimiento se visualiza también en su nueva sede principal, "de vanguardia" y mayor centro de investigación aplicada del país, según sus propulsores, con una superficie de más de 13.000 m2, 47 laboratorios, 12 plantas piloto y equipamiento de última generación. El complejo, ideado desde los inicios de Y-TEC, se encuentra operativo desde mediados de 2016 y está emplazado frente a la refinería de La Plata, en la localidad de Berisso -polo industrial de la provincia de Buenos Aires.

El formato empresarial adaptado al mercado, por otro lado, respondía a la búsqueda de vincularse estrechamente con las empresas privadas del sector, buscando lograr "competitividad" nacional e internacional. La apuesta residía no sólo en investigar, desarrollar y producir tecnologías, sino también comercializar las mismas, aportando entonces no sólo a YPF sino a toda la industria energética. Todo lo cual representaba un hito para Bianchi, dada su originalidad.

Las líneas de investigación se definirían en función de la relación con las unidades del "campo de negocios", así como también posibles servicios de asistencia técnica especializada y/o el establecimiento de consorcios. El objetivo no pasaba por tener superávit, sino crecer en nuevos equipamientos y tecnologías, y aquéllas serían las vías de financiamiento para lograrlo, generándolas por sus propios medios. Para ello era preciso atraer a las empresas siendo "serios, técnicamente excelentes" y "con alto perfil" (Salvarezza y Bianchi, 2013: 12). 
La relación entre el sistema científico argentino con el área tecnológica y la industria se leía como muy pobre, y se debía avanzar en ese sentido. Así, las figuras puestas al frente (tanto de YPF como de Y-TEC) expresaban claramente la concepción de una "gestión profesionalizada": no se optaba por cuadros políticos, con experiencia y trayectoria en cuanto a organización, conducción y planificación política, sino por cuadros técnicos/profesionales, primando la experiencia y trayectoria en el sector hidrocarburífero, y en particular el desempeño en sus grandes empresas.

\section{Acople de la CyT a la estructura productiva: ¿con qué empresas y para qué?}

Un aspecto importante en cuanto al rol que debía cumplir Y-TEC estaba orientado a la realización de actividades de formación, transferencia de conocimientos y prestación de servicios a pequeñas y medianas empresas del sector, es decir, los proveedores regionales de YPF que apostaba a consolidar el flamante Programa Sustenta. Diseñado para apuntalar la productividad y competitividad de proveedores, este Programa indicaría qué pymes tenían potencialidad pero no podían pagar un departamento de I+D, quedando excluidas del know how tecnológico requerido para el desarrollo de productos y servicios que precisaría YPF bajo su nueva gestión. En base al objetivo de venta de productos nacionales de alta calidad, aquí entraba el rol a cumplir por Y-TEC: aportar "todo el apoyo tecnológico para que saquen un producto determinado. Si ese producto es de muy buena calidad, YPF lo va a comprar y al ser de calidad se puede exportar. La pyme tiene como ventaja la compra de su producto e Y-TEC tiene un royalty en la venta" (Salvarezza y Bianchi, 2013: 12).

Sin embargo, esa función parece haber entrado en tensión con otra, en que la articulación debería darse con grandes empresas. Se aprecia una contradicción entre vías o estrategias de desarrollo disímiles, en el marco del proyecto político-económico del segundo gobierno de CFK: ¿el rol de motor del desarrollo lo ocuparía un Estado empresario, articulando pymes y ganando en funciones de control, regulación y planificación en los resortes estratégicos de la economía, como el sector hidrocarburífero? ¿O volvería a insistirse con la búsqueda de consolidar una burguesía nacional que lograra motorizar el desarrollo, como en la frustrada "argentinización" de YPF con la entrada del grupo Petersen en $2007^{3}$ ?

En este punto resulta relevante la figura de Santiago Sacerdote, Director General Adjunto de Y-TEC por parte del CONICET, desde su creación, y Gerente General de Y-TEC desde mediados de 2015 en reemplazo de Bianchi. Según se puede observar, esa figura condensaba la apuesta del último gobierno de CFK por aliarse con la Unión Industrial Argentina (UIA) y el gran empresariado local ${ }^{4}$, en el marco de la "sintonía fina" que proponía al comienzo de su segundo mandato. El camino elegido para motorizar el desarrollo, en un marco de crecientes dificultades que manifestaba el modelo de acumulación

\footnotetext{
${ }^{3}$ Ese año, el primer gobierno de CFK alentó la entrada de "capitales nacionales" a YPF a través del grupo Petersen (familia Eskenazi), el cual llegó a alcanzar el 25\% de su capital accionario. Ello no dio los resultados esperados, debido a las prácticas igualmente predatorias y cortoplacistas de la burguesía local, a la par que se agudizaba la estrategia de Repsol de fuga de utilidades al exterior y desinversión programada.

${ }^{4}$ Sobrino del banquero Manuel Sacerdote, integrante de la UIA, en 2010 se incorpora al CONICET como Vicepresidente de Asuntos Tecnológicos y miembro del Directorio en representación del sector industrial.
} 
neodesarrollista, se asentaba así en los grandes grupos económicos locales. Y ello se expresaba a su vez en las políticas referentes a YPF, con crecientes concesiones al gran empresariado del sector en busca de acompañamiento al "modelo" en cuanto a inversiones, mientras que se reestablecían vínculos con organismos internacionales de crédito en pos de conseguir financiamiento externo ${ }^{5}$ (Bilmes, 2017).

Se comprende entonces por qué el monto de los recursos asignados al Programa Sustenta no fue particularmente significativo en relación con los gastos totales de YPF-US\$ 50 millones frente a 2.800 de inversión total promedio anual entre 2012 y 2014-, a la par que esa inversión se vio canalizada por intermedio de tres de las cuatro empresas transnacionales líderes en servicios de explotación y perforación (Schlumberger, Weatherford y Halliburton, con poco más del 20\% del total cada una de ellas), que organizaron la red a partir de sus ventajas tecnológicas a nivel global. Cabe mencionar sintéticamente, a su vez, que ya con el gobierno de Mauricio Macri desde diciembre de 2015 y con Juan José Aranguren, ex CEO de Shell, al frente del nuevo Ministerio de Energía, YPF prosiguió su fuerte asociación con Schlumberger. Galuccio, en particular, luego de su salida de YPF en abril de 2016, se reincorpora en febrero de 2017 a su ex empresa, para integrarse en el directorio global de la compañía.

Por otro lado, pueden verse aspectos similares en torno a la importante cuestión del litio, una de las áreas relevantes de Y-TEC desde su creación. El desarrollo tecnológico orientado a la industrialización de ese mineral constituyó desde los inicios una de las divisiones importantes de la empresa. Esto está dado por el carácter estratégico del litio, y por el hecho de que Argentina, junto con Bolivia y Chile, cuentan con una muy importante cantidad de las reservas mundiales (alrededor del 70/85\% se hallaría en el "triángulo del litio" ubicado entre esos países). CFK destacaba este aspecto, a poco de dejar el gobierno en 2015 , señalando como un objetivo producir baterías de litio, para dejar de exportar el mineral en crudo y en cambio tener un producto con valor agregado. Con este sentido se desarroIlaron iniciativas en Jujuy para construir un Centro de Investigaciones del Litio, y se buscó conformar un consorcio público-privado para crear la primera planta de fabricación de celdas de ion-litio. Y-TEC sería la responsable de entregar la planta "llave en mano", a la par que operaría como socio tecnológico estratégico, brindando asistencia técnica, así como I+D para actualización y mejora tecnológica de los productos a elaborar. Ya con el nuevo gobierno de Cambiemos, y continuando Sacerdote en su puesto, se avanzó en ello, suscribiendo los acuerdos previos, pero ahora bajo un discurso en torno al litio como una oportunidad de negocios y no ya como un recurso estratégico en pos de la soberanía, haciendo un llamado, a tono con el discurso gubernamental, a "un actor industrial y emprendedor" para un negocio de fabricación de celdas, y potencialmente, de ensamblado de baterías. Para ello se estableció un acuerdo con la empresa italiana FIB-FAAM (SERI Group), pasando Y-TEC a trabajar con su subsidiaria Lithops en actividades de I+D para esas tecnologías.

\footnotetext{
${ }^{5}$ Convenio YPF-Chevron, acuerdo ante el CIADI (Centro Internacional de Arreglo de Diferencias relativas a Inversiones del Banco Mundial), cierre del proceso expropiatorio con pago a Repsol y acuerdo con el Club de París, entre otras medidas
} 
6

| Revista: Ciencia, Tecnología y Política | Año 1 Número 1 | www.revistas.unlp.edu.ar/CTyP |

\section{Innovación Abierta y capitalismo global}

En 2016 Y-TEC lanzó los Espacios de Innovación, definidos como plataformas de vinculación estratégica entre el sistema científico nacional y la industria energética, con el objetivo de complementar capacidades, conocimientos y equipos. A través de un conjunto de ámbitos comunes de investigación con instituciones C-T, tanto públicas como privadas (se anunciaban 7 ya concretados y la proyección de otros 30), se proponía generar productos tecnológicos de alto impacto.

Estos Espacios se enmarcaban en un modelo llamado Innovación Abierta, en auge a nivel mundial desde hace poco más de una década. En un artículo de la revista Desafíos de Y-TEC (González Ferrer, Sid y Rabal, 2016) de aquel entonces, se señala la transición en curso desde el proceso tradicional de innovación hacia ese nuevo paradigma. Así, bajo un formato "colaborativo", se buscaba permeabilizar los límites de la organización, compartiendo conocimientos y tomas de decisiones con agentes externos, tanto en el desarrollo como en la explotación de innovaciones tecnológicas, lo cual podría dar lugar a cambios estructurales en el modelo de negocios, las tecnologías y la propiedad intelectual.

Según se desprende de lo anterior, este nuevo paradigma de la innovación se corresponde con la tercerización, fragmentación e híper-especialización del trabajo a que han dado lugar las nuevas formas de producción toyotistas imperantes a nivel mundial, organizadas en redes de cadenas globales de valor "flexibles", en tiempos de un capitalismo globalizado y financiarizado (Formento, Dierckxsens y Sosa, 2017). En este marco, bajo este modelo de negocios se pretende que las empresas externalicen costos al dejar de sostener grandes áreas de I+D+i, y utilicen los conocimientos y desarrollos tecnológicos generados en universidades, institutos, laboratorios y centros de investigación, tanto públicos como privados. Una apuesta que, se ha demostrado, resulta inviable para los países del Sur Global, subordinados a los intereses de los grandes centros del poder mundial.

En este cuadro general se asienta el discurso sobre el "emprendedorismo" del gobierno de Macri, en pos de insertarse en los negocios de las cadenas globales de valor, adaptándose a los nuevos paradigmas para sostener la productividad y competitividad necesarias. Así, lejos de paralizar, subejecutar o desfinanciar los proyectos originales de Y-TEC impulsados y desarrollados en el gobierno de CFK, como se podría haber esperado de un programa neoliberal, el gobierno de Mauricio Macri ha retomado y relanzado parte importante de ellos, bajo otro marco y objetivos.

\section{Conclusiones}

El diseño y formato dado a Y-TEC por el gobierno de CFK, en sintonía con la nueva YPF, muestra que la estrategia de desarrollo propuesta dio lugar a una tensión en torno a de qué manera y con qué empresas se daría el deseado acople de la infraestructura C-T con la estructura productiva. Las empresas transnacionales y los grupos económicos locales aparecen como los beneficiarios de esa estrategia, en detrimento de la consolidación de un entramado de proveedores regionales de YPF. 
Por otro lado, ya con el gobierno de Mauricio Macri, a diferencia de lo que ocurre con otros desarrollos científico-tecnológicos, se sostiene la gestión de Y-TEC, aprovechando su estructura y articulaciones, pero reformulando sus objetivos en pos de los negocios de los sectores a los que este gobierno representa y en desmedro de los objetivos originales vinculados a la soberanía nacional.

\section{Referencias}

Bilmes, J. (2017). Nueva YPF (2012-2015): circuito hidrocarburífero, modelo de acumulación y geopolítica. Tesis de grado. UNLP.

Formento, W., Dierckxsens, W. y Sosa, M. (Marzo, 2017). "Capital financiero global, crisis, acumulación y trabajo", ponencia presentada en Congreso 50 años de Estudios Latinoamericanos en la UNAM. Teoría del valor y crisis. Universidad Nacional Autónoma de México.

González Ferrer, J. M, Sid, M. E. y Rabal, N. L. (2016). “El desafío de la Innovación Abierta”. Desafíos, \#8, pp. 54-57. Revista Desafíos (2016). “Espacios de Innovación”. \#8, pp. 58-61.

Sabato, J. y Botana, N. (2011 [1975]). La ciencia y la tecnología en el desarrollo futuro de América Latina. En Sabato, J. A. (coord.) El pensamiento latinoamericano en la problemática ciencia-tecnología-desarrollo-dependencia (pp. 215-231). Buenos Aires: Ediciones Biblioteca Nacional.

Salvarezza, R. y Bianchi, G. (2013). “La buena investigación, llave del futuro”. Desafíos, \#1, pp. 10-15. 